\title{
The NS3 protease and helicase domains of Japanese encephalitis virus trigger cell death via caspase-dependent and -independent pathways
}

\author{
GIOU-TENG YIANG ${ }^{1,2}$, YEN-HSIEH CHEN ${ }^{3}$, PEI-LUN CHOU ${ }^{4}$, \\ WEI-JUNG CHANG ${ }^{5}$, CHYOU-WEI WEI ${ }^{6}$ and YUNG-LUEN YU ${ }^{5,7}$
}

${ }^{1}$ Department of Emergency Medicine, Tzu Chi University, Hualien 970; ${ }^{2}$ Department of Emergency Medicine, Buddhist Tzu Chi General Hospital, Taipei Branch, New Taipei City 231; ${ }^{3}$ Department of Life Science, Fu Jen Catholic University, New Taipei City $242 ;{ }^{4}$ Department of Internal Medicine,

Division of Allergy-Immunology-Rheumatology, Saint Mary's Hospital, Luodong 265; ${ }^{5}$ Graduate Institute of Cancer Biology and Center for Molecular Medicine, China Medical University, Taichung $404 ;{ }^{6}$ Department of Nutrition,

Hungkuang University, Taichung 433; ${ }^{7}$ Department of Biotechnology, Asia University, Taichung, Taiwan 413, R.O.C.

Received September 4, 2012; Accepted December 4, 2012

DOI: $10.3892 / \mathrm{mmr} .2013 .1261$

\begin{abstract}
Japanese encephalitis virus (JEV), a mosquito-borne flavivirus, causes acute encephalitis and nervous damage. Previous studies have demonstrated that JEV induces apoptosis in infected cells. However, to date the mechanisms of JEV-induced apoptosis are unclear. In order to identify the viral proteins associated with JEV-induced apoptosis, pEGFP-non-structural protein 3 (NS3) 1-619 (expressing the JEV NS3 intact protein, including the protease and helicase domains), pEGFP-NS3 1-180 (expressing the protease domain) and pEGFP-NS3 163-619 (expressing the helicase domain) were transfected into target cells to study cell death. Results demonstrate that the JEV NS3 intact protein and protease and helicase domains induce cell death. In addition, cell death was identified to be significantly higher in cells transfected with the NS3 protease domain compared with the intact protein and helicase domain. Caspase activation was also analyzed in the current study. NS3 intact protein and NS3 protease and helicase domains activated caspase-9/-3-dependent and -independent pathways. However, caspase- 8 activity was not found to be significantly different in NS3-transfected cells compared with control. In summary, the present study demonstrates that the NS3 helicase and protease
\end{abstract}

Correspondence to: Dr Yung-Luen Yu, Graduate Institute of Cancer Biology, China Medical University, 6 Hsueh-Shih Road Taichung 404, Taiwan, R.O.C.

E-mail: ylyu@mail.cmu.edu.tw

Dr Chyou-Wei Wei, Department of Nutrition, Master Program of Biomedical Nutrition, Hungkuang University, 1018 Sec. 6, Taiwan Boulevard, Taichung 433, Taiwan, R.O.C.

E-mail:wcwnina@gate.sinica.edu.tw

Key words: Japanese encephalitis virus, helicase, protease, apoptosis, caspase domains of JEV activate caspase-9/-3-dependent and -independent cascades and trigger cell death.

\section{Introduction}

Japanese encephalitis virus (JEV) is a member of the Flaviviridae family and infection is associated with nervous damage and encephalitis (1-3). Previous studies have demonstrated that JEV infection causes cell death via the apoptotic pathway $(4,5)$. In addition, infection increases oxidative stress and activates ERK/p38 MAPK signal transduction $(6,7)$. $\mathrm{JEV}$-induced apoptosis via caspase activation has been previously reported (8-10). However, the component of JEV which induces cell death remains unclear.

Similar to JEV, dengue virus type 2 (DENV), hepatitis C virus (HCV), West Nile virus (WNV) and langat flavivirus are all members of the Flaviviridae family (11-14). These viruses cause cell death in infected cells, although their clinical presentation varies (15-18). To date, a number of studies have reported that induction of cell death by DENV, HCV, WNV and langat flavivirus may be associated with non-structure protein 3 (NS3) domains present in the viruses (19-22). In addition, previous studies have demonstrated that JEV NS2B-NS3 fragments activate caspase- 3 and induce apoptosis in human medulloblastoma cells (23). Based on these observations, we hypothesized that the JEV NS3 protein may be important for JEV-induced apoptosis. Therefore, to examine whether JEV NS3 proteins induce cell apoptosis, pEGFP-NS3 1-619 (expressing the NS3 intact protein) was transfected into cells. Results indicate that the JEV NS3 protein induces apoptotic cell death.

The JEV NS3 protein has important enzyme activities, including helicase, protease and nucleoside triphosphatase activities (24-26). Previous studies have cloned C-terminal residues of JEV NS3 and identified RNA helicase activity (27). In addition, a number of studies have cloned $\mathrm{N}$-terminal residues of NS3 using a PCR method, identifying protease 
activity (28-30). In order to examine which fragments of the JEV NS3 protein induce cell apoptosis, pEGFP-NS3 163-619 or pEGFP-NS3 1-180 (expressing the NS3 helicase and protease domains, respectively), were transfected into cells and cell death percentage was calculated. The current study demonstrates that the NS3 helicase and protease domains induce cell death in a manner comparable with the JEV NS3 intact protein.

Cell death is divided into two pathways, necrosis and apoptosis (31). In the apoptosis pathway, chromatin condensation and DNA fragmentation are observed $(32,33)$ and the process is mediated by caspase cascades $(34,35)$. Two major caspase cascades have been reported, including death receptor and mitochondria-mediated death pathways (36). In the death receptor pathway, initiator caspase- 8 and executioner caspase- 3 are activated to induce cell apoptosis through death ligands, which bind cell surface death receptors (37). In the mitochondrial death pathway, initiator caspase- 9 and executioner caspase- 3 are activated to mediate cell apoptosis via mitochondrial dysfunction (38). In this study, caspase-9 and -3 were activated in cells transfected with pEGFP-NS3 1-619, pEGFP-NS3 163-619 and pEGFP-NS3 1-180. However, caspase-8 activity was unchanged in groups compared with the control. Overall, results demonstrate that JEV NS3 and the NS3 protease and helicase domains induce cell death via the caspase-9/-3 cascade pathway.

\section{Materials and methods}

Plasmids and chemicals. pEGFP (expressing green fluorescent protein), pEGFP-NS31-619 (expressing NS3 intact protein), pEGFP-NS3 1-180 (expressing NS3 protease domain) and pEGFP-NS3 163-619 ( expressing NS3 helicase domain) were kindly donated by Dr Jaang-Jiun Wang (Division of Pediatric Infectious Diseases, Emory University School of Medicine, Atlanta, USA). Acetyl-Asp-Glu-Val-Asp-p-nitroanilide (Ac-DEVD-pNA; caspase-3 substrate), Ac-Leu-Glu-His-Asp (LEHD)-pNA (caspase-9 substrate) and Ac-Ile-Glu-Thr-Asp (IETD)-pNA (caspase-8 substrate) were obtained from Anaspec (Fremont, CA, USA). Gene Jammer ${ }^{\circledR}$ transfection reagent was purchased from Agilent Technologies (Santa Clara, CA, USA). Fetal bovine serum (FBS) was obtained from Invitrogen Life Technologies (Carlsbad, CA, USA). DMEM, non-essential amino acid, L-glutamine and penicillin/streptomycin were obtained from Gibco-BRL (Carlsbad, CA, USA).

Cell lines and cell culture. Vero (green monkey kidney epithelial) and HeLa cells (human cervical cancer) were obtained from the Bioresources Collection and Research Center (Hsin Chu, Taiwan). Cells were cultured in DMEM supplemented with $10 \%$ heat-inactivated FBS, 2 mM L-glutamine, 100 IU/ml penicillin/streptomycin and $0.1 \mathrm{mM}$ non-essential amino acids and maintained at $37^{\circ} \mathrm{C}$ in a humidified atmosphere containing $5 \% \mathrm{CO}_{2}$. The study was approved by the ethics committee of Graduate Institute of Cancer Biology, China Medical University, Taichung, Taiwan.

Transfection. Vero and Hela cells were incubated with $2 \mu \mathrm{g}$ plasmid (pEGFP, pEGFP-NS3 1-619, pEGFP-NS3 1-180 and pEGFP-NS3 163-619) mixed with $6 \mu$ l Gene Jammer ${ }^{\circledR}$ transfection reagent and added to $1 \mathrm{ml}$ DMEM, in 6-well plates
( $2 \times 10^{5}$ cells/well) for 3 hours. Following this, the medium was replaced with DMEM supplemented with $10 \%$ heat-inactivated FBS, 2 mM L-glutamine, $100 \mathrm{IU} / \mathrm{ml}$ penicillin/streptomycin and $0.1 \mathrm{mM}$ non-essential amino acids and maintained at $37^{\circ} \mathrm{C}$ in a humidified atmosphere containing 5\% $\mathrm{CO}_{2}$. Following $16 \mathrm{~h}$ transfection, the plasmid expressed the gene products.

Cell death percentage assay. Cells $\left(1 \times 10^{5}\right)$ were grown in a 24 -well plate. Following $24 \mathrm{~h}$ plasmid transfection, cell number was calculated using the trypan blue exclusion method and a hemocytometer. Percentage of dead cells was calculated using the following formula: (100 - no. of cells in plasmid transfected group/no. of cells in plasmid non-transfected group x 100 .

Nuclear staining. Nuclear staining was performed by Hoechst 33342 as described previously (39). In brief, cells were treated with Hoechst $33342(10 \mu \mathrm{g} / \mathrm{ml})$ for $10 \mathrm{~min}$. Following this, DNA condensation and fragmentation were observed under a fluorescence microscope (excitation, $352 \mathrm{~nm}$; emission, $450 \mathrm{~nm}$ ).

Caspase activity assay. Caspase activity was determined using a substrate cleavage assay as described previously $(39,40)$. Briefly, cells were lysed with lysis buffer $(50 \mathrm{mM}$ Tris- $\mathrm{HCl}$, $120 \mathrm{mM} \mathrm{NaCl}, 1 \mathrm{mM}$ EDTA and 1\% NP-40; pH 7.5) in the presence of protease inhibitors. Cell pellets were discarded following centrifugation at $15,000 \mathrm{x} \mathrm{g}$ for $30 \mathrm{~min}$ at $4^{\circ} \mathrm{C}$. The substrate cleavage assay was executed in a working reaction containing $40 \mu \mathrm{l}$ cell lysate (80 $\mu \mathrm{g}$ total protein), $158 \mu \mathrm{l}$ reaction buffer (20\% glycerol, $0.5 \mathrm{mM}$ EDTA, $5 \mathrm{mM}$ dithiothreitol and $100 \mathrm{mM}$ HEPES; $\mathrm{pH} 7.5$ ) and $2 \mu \mathrm{l}$ fluorogenic substrate (either Ac-LEHD-pNA, Ac-IETD-pNA or Ac-DEVD-pNA; $100 \mu \mathrm{M}$ final concentration). The reaction was incubated at $37^{\circ} \mathrm{C}$ for $6 \mathrm{~h}$. Cleavage of the fluorogenic substrate released p-NA, which was measured at $405 \mathrm{~nm}$ in an ultra-microplate reader (Ceres UV 900, BioTek Instruments, Inc., Winooski, VT, USA). Fold increase in caspase activity was calculated using the following formula: (A405 sample $-\mathrm{A} 405_{\text {control }}$ )/A405 $5_{\text {control }}$.

Inhibition of caspase activity. Z-VAD-FMK is a general caspase inhibitor which inhibits all caspase activity. Inhibition of caspase activity was performed as described previously (39). Briefly, cells were pretreated with $10 \mathrm{mM}$ Z-VAD-FMK prior to transfection. Following this, the percentage of dead cells was determined as described for cell death percentage assay.

Statistical analysis. Data were obtained from four independent triplicate experiments and are presented as the mean values of the representative triplicate experiment (mean \pm SD). Statistical differences between 2 groups were determined by the Student's t test.

\section{Results}

NS3 intact protein induces cell death through the apoptosis pathway. Plasmid pEGFP expresses a green fluorescent protein and plasmid pEGFP-NS3 1-619 expresses the JEV NS3 intact protein. Following transfection of Vero cells with pEGFP or pEGFP-NS3 1-619 for 24 hours, cell counts were performed and the percentage of dead cells was calculated. 


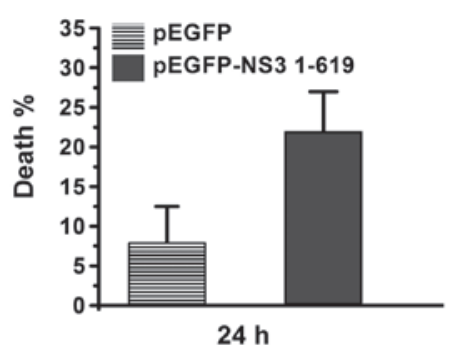

Figure 1. Trypan blue exclusion analysis at $24 \mathrm{~h}$ following Vero cell transfection with pEGFP or pEGFP-NS3 1-619. Percentage of dead cells was calculated. Data were obtained from four independent experiments and presented as mean \pm SD. NS3, non-structure protein 3; JEV, Japanese encephalitis virus.

As demonstrated in Fig. 1, percentage of dead cells was $\sim 8 \%$ in the pEGFP-transfected group. However, the percentage of dead cells was $>20 \%$ in the pEGFP-NS3 1-619-transfected group, indicating that JEV NS3 induces cell death. Next, nuclear morphology was analyzed in transfected cells via the Hoechst 33342 staining method. Compared with pEGFP-transfected cells (Fig. 2A), chromatin condensation and DNA fragmentation were found in pEGFP-NS3 1-619-transfected cells (Fig. 2B). Results indicate that the JEV NS3 intact protein induces cell death through the apoptosis pathway.

NS3 helicase and protease domains induce cell death. JEV NS3 intact protein includes two major domains, the protease and helicase domains. pEGFP-NS3 1-180 and pEGFP-NS3 163-619 (expressing the JEV NS3 protease and helicase domains, respectively) were used in this study. The percentage of dead cells was determined $24 \mathrm{~h}$ after transfection of Vero cells with pEGFP, pEGFP-NS3 1-619, pEGFP-NS3 1-180 or pEGFP-NS3 163-619 (Fig. 3). In the pEGFP-transfected group, cell death was $\sim 6 \%$. In the pEGFP-NS3 1-619 and pEGFP-NS3 163-619-transfected groups, cell death was $20 \%$ and in the pEGEP-NS3 1-180-transfected group, cell death was $\sim 30 \%$. These results demonstrate that cell death is induced by the JEV NS3 intact protein as well as the the JEV NS3 helicase and protease domains. In addition, the protease domain was observed to exert a higher cytotoxic effect compared with the intact protein and helicase domain.

JEV NS3 intact protein and helicase and protease domains induce the caspase-9/-3 cascade pathway. Caspase activities were also determined in the current study, following $24 \mathrm{~h}$ transfection of Vero cells with pEGFP, pEGFP-NS3 1-619, pEGFP-NS3 1-180 or pEGFP-NS3 163-619 (Fig. 4). Compared with the pEGFP-transfected group, caspase-3 and -9 were markedly activated in pEGFP-NS3 1-619, pEGFP NS31-180 and pEGFP-NS3 163-619-transfected groups (Fig. 4A and B). However, compared with pEGFP, caspase-8 was not found to be significantly activated in pEGFP-NS3 1-619, pEGFP-NS3 1-180 and pEGFP-NS3 163-619-transfected groups (Fig. 4C). Results indicate that the JEV NS3 intact protein and protease and helicase domains activate the caspase-9/-3 cascade pathway.

JEV NS3 protein and protease and helicase domains induce cell death through caspase-independent pathways. To determine whether JEV NS3 intact protein- and protease and helicase domain-induced cell death is dependent on caspase activity, Vero cells were treated with Z-VA-FMK (a general
A
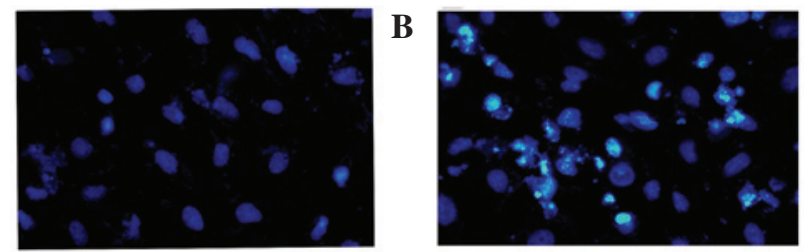

Figure 2. Hoechst 33342 nulear staining for analysis of nuclear morphology (A) pEGFP- and (B) pEGFP-NS3 1-619-transfected cells. Chromatin condensation and DNA fragmentation was observed in pEGFP-NS3 1-619-transfected cells, indicating that JEV NS3 intact protein induces apoptosis. NS3, non-structure protein 3; JEV, Japanese encephalitis virus.

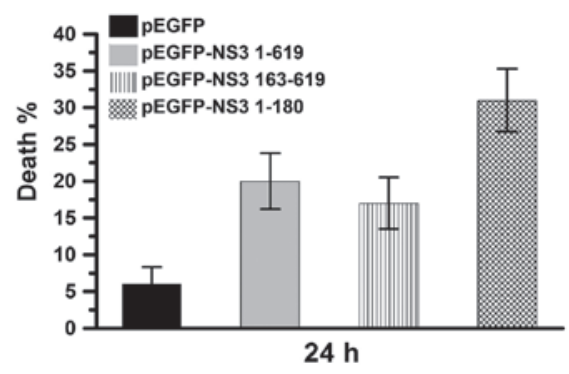

Figure 3. Trypan blue exclusion analysis at $24 \mathrm{~h}$ following transfection of Vero cells with pEGFP, pEGFP-NS3 1-619, pEGFP-NS3 163-619 or pEGFP-NS3 1-180. The percentage of dead cells was calculated. Data were obtained from four independent experiments and presented as mean \pm SD. NS3 helicase and protease domains of JEV induced cell death. NS3, non-structure protein 3; JEV, Japanese encephalitis virus.
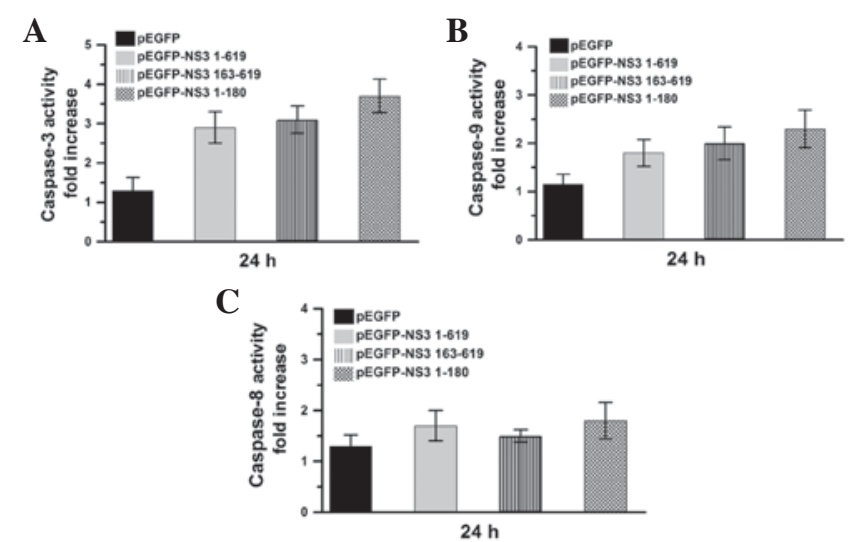

Figure 4. Caspase activity assay. Activities of (A) caspase-3, (B) -9 and (C) -8 were determined in Vero cells transfected with pEGFP, pEGFP-NS3 1-619, pEGFP-NS3 163-619 and pEGFP-NS3 1-180. Caspase-3 and -9 activity significantly increased in the pEGFP-NS3 1-619-, pEGFP-NS3 163-619and pEGFP-NS3 1-180-transfected groups, indicating that the JEV NS3 intact protein and helicase and protease domains induce cell death via a caspase-9/-3-dependent pathway. Data were obtained from three independent experiments and presented as mean \pm SD. NS3, non-structure protein 3; JEV, Japanese encephalitis virus.

caspase inhibitor) prior to plasmid transfection and cell death percentage was calculated. As demonstrated in Fig. 5, cell death was $<10 \%$ in pEGFP-, $>15 \%$ in pEGFP-NS3 1-619- and pEGFP-NS3 163-619- and $25 \%$ in pEGFP-NS3 1-180-transfected groups. Therefore, the NS3 intact protein and protease and helicase domains may also induce cell death by caspase-independent pathways. In summary, this study revealed that the JEV NS3 intact protein and protease and NS3 


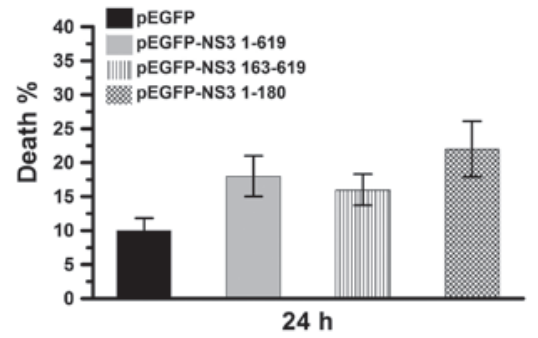

Figure 5. Trypan blue exclusion analysis. Vero cells were treated with caspase inhibitor, Z-VAD-FMK, for $30 \mathrm{~min}$, followed by transfection with pEGFP, pEGFP-NS3 1-619, pEGFP-NS3 163-619 or pEGFP-NS3 1-180. The percentage of dead cells was calculated. Data were obtained from four independent experiments and presented as mean \pm SD. JEV NS3 intact protein and helicase and protease domains induced cell death via a caspase-independent pathway. NS3, non-structure protein 3; JEV, Japanese encephalitis virus.

helicase domains induce cell death via caspase-9/-3 cascade and caspase-independent pathways.

\section{Discussion}

A number of previous studies have demonstrated that JEV infection induces cell death via a caspase-dependent cell death pathway $(4,8-10,41)$. However, the involvement of various JEV proteins with cell death remains unclear. A previous study indicated that JEV NS2-NS3 fragments induce cell death (23). Consistent with these observations, the current study demonstrates that the JEV NS3 protein alone is sufficient to trigger cell death (Fig. 1). JEV NS3 protein has been previously identified to exhibit protease and helicase enzyme activities at the $\mathrm{N}$ - and C-terminals, respectively $(25,42,43)$. JEV NS3 protein is known to induce cell death; however, the enzyme activities associated with this process are not known and were analyzed in the present study. Results demonstrate that the protease and helicase domains alone trigger cell death (Fig. 3), indicating that these activities induce cell death following JEV infection.

Caspase-dependent death pathways include the caspase-8/-3 and $-9 /-3$ cascade pathways (36-38). The caspase-8/-3 death pathway is induced by ligands acting on death receptors located in the cell membrane $(37,44)$. However, the caspase-9/-3 death pathway is activated by mitochondrial dysfunction $(38,44)$. Previous studies have reported that JEV infection triggers activation of caspase-3, -8 and $-9(10,41)$, indicating that JEV infection induces cell death through the death receptor and mitochondrial pathways. However, the present results indicate that the JEV NS3 protein and protease and helicase domains activate caspase- 9 and -3 activity only and do not activate caspase-8 (Fig. 4). Therefore, these fragments induce the caspase-9/-3 mitochondrial death pathway only. To understand why the death receptor pathway is activated in JEV-infected cells but not in JEV NS3-, protease- and helicase-expressed cells, we hypothesized that the virus enters the cell across the membrane and the viral capsid may interact with death receptors leading to activation of the caspase-8/-3 death pathway during JEV infection. The caspase-9/-3 pathway is not activated until later in the infection process when NS3 protein is expressed in cells. Therefore, results indicate that the JEV NS3 protein and protease and helicase domains markedly induce the caspase- $9 /-3$ but not the caspase- $8 /-3$ cascades .
Numerous studies have demonstrated that cell death is induced through the caspase-dependent and -independent pathways (45-48). JEV infection is known to induce cell death through a caspase-dependent pathway $(10,23)$; however, studies have not determined whether JEV-induced cell death is also associated with caspase-independent pathways. The present results demonstrate that inhibition of caspase activity, using the general caspase inhibitor Z-VAD-FMK $(39,49)$, did not prevent cell death in pEGFP-NS3 1-619-, pEGFP-NS3 1-180- and pEGFP-NS3 613-619-transfected cells (Fig. 5), indicating that these fragments induce cell death through a caspase-independent pathway. Recent studies have demonstrated that a number of compounds, including isoegomaketone, transglutaminase 2 and blazeispirol A, induce cell death through caspase-dependent and -independent pathways (50-52). Consistent with these observations, the current study indicates that the JEV NS3 protein, as well as the JEV NS3 protease and helicase domains induce cell death through caspase-dependent (caspase-9/-3 cascade) and -independent pathways (Figs. 4 and 5).

\section{Acknowledgements}

The present study was supported by grants from the National Science Council of Taiwan (NSC99-2320-B-039-030-MY3, NSC99-2632-B-039-001-MY3 and NSC100-2321-B-039-004) and The University of Texas MD Anderson-China Medical University and Hospital Sister Institution Fund (DMR-101-115).

\section{References}

1. Hall-Mendelin S, Jansen CC, Cheah WY, et al: Culex annulirostris (Diptera: Culicidae) host feeding patterns and Japanese encephalitis virus ecology in northern Australia. J Med Entomol 49: 371-377, 2012.

2. Srivastava R, Kalita J, Khan MY and Misra UK: Status of proinflammatory and anti-inflammatory cytokines in different brain regions of a rat model of Japanese encephalitis. Inflamm Res 61: 381-389, 2012.

3. Xufang Y, Huanyu W, Shihong F, et al: Etiological spectrum of clinically diagnosed Japanese encephalitis cases reported in Guizhou Province, China, in 2006. J Clin Microbiol 48: 1343-1349, 2010.

4. Liao CL, Lin YL, Wang JJ, et al: Effect of enforced expression of human bcl-2 on Japanese encephalitis virus-induced apoptosis in cultured cells. J Virol 71: 5963-5971, 1997.

5. Liao CL, Lin YL, Shen SC, et al: Antiapoptotic but not antiviral function of human bcl-2 assists establishment of Japanese encephalitis virus persistence in cultured cells. J Virol 72: 9844-9854, 1998.

6. Tung WH, Tsai HW, Lee IT, et al: Japanese encephalitis virus induces matrix metalloproteinase- 9 in rat brain astrocytes via NF-kappaB signalling dependent on MAPKs and reactive oxygen species. Br J Pharmacol 161: 1566-1583, 2010.

7. Yang TC, Lai CC, Shiu SL, et al: Japanese encephalitis virus down-regulates thioredoxin and induces ROS-mediated ASK1-ERK/p38 MAPK activation in human promonocyte cells. Microbes Infect 12: 643-651, 2010.

8. Gupta N, Bhaskar AS and Lakshmana Rao PV: Transcriptional regulation and activation of the mitogen-activated protein kinase pathway after Japanese encephalitis virus infection in neuroblastoma cells. FEMS Immunol Med Microbiol 62: 110-121, 2011.

9. Lee YH, Wei CW, Wang JJ and Chiou CT: Rana catesbeiana ribonuclease inhibits Japanese encephalitis virus (JEV) replication and enhances apoptosis of JEV-infected BHK-21 cells. Antiviral Res 89: 193-198, 2011.

10. Tsao CH, Su HL, Lin YL, et al: Japanese encephalitis virus infection activates caspase- 8 and -9 in a FADD-independent and mitochondrion-dependent manner. J Gen Virol 89: 1930-1941, 2008. 
11. Markoff LJ, Innis BL, Houghten R and Henchal LS: Development of cross-reactive antibodies to plasminogen during the immune response to dengue virus infection. J Infect Dis 164: 294-301, 1991.

12. Fletcher NF and McKeating JA: Hepatitis $\mathrm{C}$ virus and the brain J Viral Hepat 19: 301-306, 2012.

13. Osorio HC, Ze-Ze L and Alves MJ: Host-feeding patterns of Culex pipiens and other potential mosquito vectors (Diptera: Culicidae) of West Nile virus (Flaviviridae) collected in Portugal. J Med Entomol 49: 717-721, 2012.

14. Iacono-Connors LC and Schmaljohn CS: Cloning and sequence analysis of the genes encoding the nonstructural proteins of Langat virus and comparative analysis with other flaviviruses. Virology 188: 875-880, 1992

15. Liao $\mathrm{H}, \mathrm{Xu} \mathrm{J}$ and Huang J: FasL/Fas pathway is involved in dengue virus induced apoptosis of the vascular endothelial cells. J Med Virol 82: 1392-1399, 2010.

16. Korner C, Tolksdorf F, Riesner K, et al: Hepatitis C coinfection enhances sensitization of CD4(+) T-cells towards Fas-induced apoptosis in viraemic and HAART-controlled HIV-1-positive patients. Antivir Ther 16: 1047-1055, 2011.

17. Kumar M, Verma S and Nerurkar VR: Pro-inflammatory cytokines derived from West Nile virus (WNV)-infected SK-N-SH cells mediate neuroinflammatory markers and neuronal death. J Neuroinflammation 7: 73, 2010.

18. Prikhod'ko GG, Prikhod'ko EA, Cohen JI and Pletnev AG: Infection with Langat Flavivirus or expression of the envelope protein induces apoptotic cell death. Virology 286: 328-335, 2001.

19. Shafee N and AbuBakar S: Dengue virus type 2 NS3 protease and NS2B-NS3 protease precursor induce apoptosis. J Gen Virol 84: 2191-2195, 2003

20. Gamlen T, Richards KH, Mankouri J, et al: Expression of the NS3 protease of cytopathogenic bovine viral diarrhea virus results in the induction of apoptosis but does not block activation of the beta interferon promoter. J Gen Virol 91: 133-144, 2010.

21. Ramanathan MP, Chambers JA, Pankhong P, et al: Host cell killing by the West Nile Virus NS2B-NS3 proteolytic complex: NS3 alone is sufficient to recruit caspase-8-based apoptotic pathway. Virology 345: 56-72, 2006.

22. Prikhod'ko GG, Prikhod'ko EA, Pletnev AG and Cohen JI: Langat flavivirus protease NS3 binds caspase- 8 and induces apoptosis. J Virol 76: 5701-5710, 2002.

23. Yang TC, Shiu SL, Chuang PH, et al: Japanese encephalitis virus NS2B-NS3 protease induces caspase 3 activation and mitochondria-mediated apoptosis in human medulloblastoma cells. Virus Res 143: 77-85, 2009.

24. Yamashita T, Unno H, Mori Y, et al: Crystal structure of the catalytic domain of Japanese encephalitis virus NS3 helicase/nucleoside triphosphatase at a resolution of $1.8 \mathrm{~A}$. Virology 373: 426-436, 2008.

25. Borowski P, Heising MV, Miranda IB, Liao CL, Choe J and Baier A: Viral NS3 helicase activity is inhibited by peptides reproducing the Arg-rich conserved motif of the enzyme (motif VI). Biochem Pharmacol 76: 28-38, 2008.

26. Lin CW, Huang HD, Shiu SY, et al: Functional determinants of NS2B for activation of Japanese encephalitis virus NS3 protease. Virus Res 127: 88-94, 2007.

27. Utama A, Shimizu H, Morikawa S, et al: Identification and characterization of the RNA helicase activity of Japanese encephalitis virus NS3 protein. FEBS Lett 465: 74-78, 2000.

28. Bera AK, Kuhn RJ and Smith JL: Functional characterization of cis and trans activity of the Flavivirus NS2B-NS3 protease. J Biol Chem 282: 12883-12892, 2007.

29. Chang YS, Liao CL, Tsao CH, et al: Membrane permeabilization by small hydrophobic nonstructural proteins of Japanese encephalitis virus. J Virol 73: 6257-6264, 1999.

30. Lin CW, Lin KH, Lyu PC and Chen WJ: Japanese encephalitis virus NS2B-NS3 protease binding to phage-displayed human brain proteins with the domain of trypsin inhibitor and basic region leucine zipper. Virus Res 116: 106-113, 2006

31. Haeri M, Wöllert T, Langford GM and Gilbert JL: Electrochemical control of cell death by reduction-induced intrinsic apoptosis and oxidation-induced necrosis on CoCrMo alloy in vitro. Biomaterials 33: 6295-6304, 2012.

32. La Vignera S, Condorelli R, Vicari E, D'Agata $R$ and Calogero AE: Effects of varicocelectomy on sperm DNA fragmentation, mitochondrial function, chromatin condensation and apoptosis. J Androl 33: 389-396, 2012
33. Burattini S, Ferri P, Battistelli M, et al: Apoptotic DNA fragmentation can be revealed in situ: an ultrastructural approach Microsc Res Tech 72: 913-923, 2009.

34. Mu S, Tian X, Ruan Y, et al: Diosgenin induces apoptosis in IGF-1-stimulated human thyrocytes through two caspase-dependent pathways. Biochem Biophys Res Commun 418: 347-352, 2012

35. Pan TL, Wang PW, Leu YL, Wu TH and Wu TS: Inhibitory effects of Scutellaria baicalensis extract on hepatic stellate cells through inducing $\mathrm{G} 2 / \mathrm{M}$ cell cycle arrest and activating ERK-dependent apoptosis via Bax and caspase pathway. J Ethnopharmacol 139: 829-837, 2012.

36. Lee CS, Kwak SW, Kim YJ, et al: Guanylate cyclase activator YC-1 potentiates apoptotic effect of licochalcone A on human epithelial ovarian carcinoma cells via activation of death receptor and mitochondrial pathways. Eur J Pharmacol 683: 54-62, 2012.

37. Yang C, Liu HZ and Fu ZX: PEG-liposomal oxaliplatin induces apoptosis in human colorectal cancer cells via Fas/FasL and caspase-8. Cell Biol Int 36: 289-296, 2012.

38. Hua YY, Wang XS, Zhang Y, Yao CG, Zhang XM and Xiong ZA Intense picosecond pulsed electric fields induce apoptosis through a mitochondrial-mediated pathway in HeLa cells. Mol Med Rep 5: 981-987, 2012.

39. Yiang GT, Yu YL, Chou PL, et al: The cytotoxic protein can induce autophagocytosis in addition to apoptosis in MCF-7 human breast cancer cells. In Vivo 26: 403-409, 2012

40. Tang $\mathrm{CH}, \mathrm{Hu} \mathrm{CC}$, Wei CW and Wang JJ: Synergism of Rana catesbeiana ribonuclease and IFN-gamma triggers distinct death machineries in different human cancer cells. FEBS Lett 579: 265-270, 2005

41. Lee CJ, Liao CL and Lin YL: Flavivirus activates phosphatidylinositol 3-kinase signaling to block caspase-dependent apoptotic cell death at the early stage of virus infection. J Virol 79: 8388-8399, 2005

42. Deng X, Shi Z, Li S, et al: Characterization of nonstructural protein 3 of a neurovirulent Japanese encephalitis virus strain isolated from a pig. Virol J 8: 209, 2011.

43. Kaczor A and Matosiuk D: Structure-based virtual screening for novel inhibitors of Japanese encephalitis virus NS3 helicase/nucleoside triphosphatase. FEMS Immunol Med Microbiol 58: 91-101, 2010.

44. Wei CW, Hu CC, Tang CH, Lee MC and Wang JJ: Induction of differentiation rescues HL-60 cells from Rana catesbeiana ribonuclease-induced cell death. FEBS Lett 531: 421-426, 2002.

45. Yu HY, Jin CY, Kim KS, et al: Oleifolioside A mediates caspase-independent human cervical carcinoma HeLa cell apoptosis involving nuclear relocation of mitochondrial apoptogenic factors AIF and EndoG. J Agric Food Chem 60: 5400-5406, 2012.

46. Jang EH, Park CS and Kang JH: Bupropion, an atypical antidepressant, induces endoplasmic reticulum stress and caspase-dependent cytotoxicity in SH-SY5Y cells. Toxicology 285: 1-7, 2011.

47. Zhi B, Tang W and Zhang X: Enhancement of shrimp antiviral immune response through caspase-dependent apoptosis by small molecules. Mar Biotechnol (NY) 13: 575-583, 2011.

48. Linsenbardt AJ, Breckenridge JM, Wilken GH and Macarthur H: Dopaminochrome induces caspase-independent apoptosis in the mesencephalic cell line, MN9D. J Neurochem 122: 175-184, 2012.

49. Furre IE, Møller MT, Shahzidi S, Nesland JM and Peng Q: Involvement of both caspase-dependent and -independent pathways in apoptotic induction by hexaminolevulinate-mediated photodynamic therapy in human lymphoma cells. Apoptosis 11: 2031-2042, 2006

50. Cho BO, Jin CH, Park YD, et al: Isoegomaketone induces apoptosis through caspase-dependent and caspase-independent pathways in human DLD1 cells. Biosci Biotechnol Biochem 75: 1306-1311, 2011

51. Yoo JO, Lim YC, Kim YM and Ha KS: Transglutaminase 2 promotes both caspase-dependent and caspase-independent apoptotic cell death via the calpain/Bax protein signaling pathway. J Biol Chem 287: 14377-14388, 2012.

52. Su ZY, Tung YC, Hwang LS and Sheen LY: Blazeispirol A from Agaricus blazei fermentation product induces cell death in human hepatoma Hep 3B cells through caspase-dependent and caspase-independent pathways. J Agric Food Chem 59: 5109-5116, 2011 